\title{
Sexually \\ Transmitted \\ Infections
}

\section{Editorials}

\section{Choosing and using services for sexual health: women's views}

As the paper on chlamydia screening by Dixon-Woods et al in this issue (p 335) shows, qualitative research can tell you how patients are referred to sexual health services and why they come. These provide compelling reasons for integrating qualitative with more standard quantitative methods in STI research. The control of infection depends not only on effective prevention and treatment of pathogens but also on the interactions between pathogen, host, and environment. Qualitative methodologies are integral to our understanding of at least two points in this triangle. Interview, fieldwork, and a range of other methods allow us to describe what people say and what they do, and to investigate the often complex relationships between the two.

The paper highlights findings from interviews with patients that are relevant to clinical and public health planning. The authors show that patients are "ambassadors" of the services and thus underscore the importance of lay rather than professional networks of referral to services. They demonstrate the importance of being able to phone for results and thus the need for patients to retain an element of control over interactions in the clinic. They show too that consultations are prompted less by concerns about a specific infection and more by a range of symptoms or behavioural cues that could fit with a number of infections, a generic "STI" as it were.

The major objection to qualitative research has generally been one of scale. STI clinic staff and scholars from other traditions may already "know" the findings of studies such as the ones reported in this issue; they seem to be mere common sense. Doctors, for example, already know that the stigma attached to clinics deters potential patients and that the fragmentation of services makes optimal management impossible, for example, in providing contraception alongside STI treatment. On the other hand, qualitative studies often also cause surprise and disbelief. In such situations, findings may be rejected above all for their lack of power: "the numbers were too small." This problem has been addressed by treating the qualitative as a pilot study leading to larger scale investigations. For example, the relevance of health promotion for a generic, and possibly asymptomatic, STI as suggested by Dixon-Woods et al would need testing in a larger population. Yet, larger studies are not always necessary. It should be possible to introduce telephoning for results and then assess the benefits for some or all patients without further research.

A second standard method of dealing with issues of scale is to integrate qualitative and quantitative research more thoroughly. Research on sexuality requires particular sensitivity and large scale surveys have drawn upon initial contextual studies showing what can sensibly be asked and analysed in terms of what are often called "knowledge, attitudes, beliefs, and practices" (KABP). They have also drawn on subsequent fieldwork and interview to help interpret data. ${ }^{1}$ A good example of combined qualitative and quantitative research is found in partner notification for STI. Recent studies in the United States have reported that sexual contact tracing provides a low yield in contrast with social contact tracing, most likely because it is hard to identify and contact all sexual partners on the one hand while, on the other, these partners are found largely among social groups of peers. ${ }^{2}$ Research combining qualitative and quantitative techniques inside clinics, through interviews of social/sexual contacts and fieldwork at places where "high risk" individuals congregate has been combined in order to better understand transmission dynamics and design interventions. In research on risks of gonorrhoea, we tested a biological marker for unique sexual networks, differentiating types of gonorrhoea, ${ }^{3}$ so as to see whether it would be possible to generalise the results of in-depth interviews and fieldwork through potentially routine clinic data.

In STI research, the qualitative has further connotations. Two of the more important include the settings that are studied and the definitions of health employed. The study of Dixon-Woods and colleagues took place in an STI clinic but many have been conducted outside the clinic and often with marginal and deprived groups, sometimes known as the "hard to reach" or "out of reach" because they do not use health services. Secondly, qualitative research tends to involve a broad social definition of health, compared with a medical view of dysfunction. This may redress current biases towards overly technological understandings and provide more appropriate bases for health promotion as patients may be concerned with one infection only in the context of others, with STI in relation to reproductive health, or with sexual health in relation to general wellbeing. In research on sex work, we have attempted to combine qualitative and quantitative elements over several years so as to build up relationships with a stigmatised social group inside and outside the clinic and develop services considered appropriate by participants, based on this more holistic model of health. In developing a "quantified anthropology" and a "social epidemiology," we also intended to provide insight into the content and meaning of activities associated with STI alongside objective outcome measures. Accordingly, neither the qualitative nor the quantitative element stands alone. If, for example, $20 \%$ of a "high risk" category do not use condoms all the time, it is necessary to 
understand when condoms are not used, among whom, and why before it is possible to design a feasible intervention. ${ }^{4}$

It should not be forgotten that the scale of qualitative research is also its strength, enabling an understanding of interactions between host, pathogen, and environment over time and in the context of relevant variables such as relationships with sexual partners and service use. These advantages tend to be lost in studies of large numbers and so it is preferable to employ one of the analytic techniques available for generalising findings about social relationships concerning class, network, sex, and so forth to "scale up" findings of this kind. Further development of these markers will make it possible to achieve greater integration between complementary studies of the biological and social, the qualitative and the quantitative.

Anthropology, Goldsmith's College, London SE14 6NW, UK and

SOPHIE DAY

Department of Epidemiology and Public Health, Imperial College School

of Medicine at St Mary's, London W2 1PG, UK

1 Van de Ven P, Aggleton P. What constitutes evidence in HIV/AIDS education? Health Educ Res 1999;14:461-71.

2 Rothenberg R, Kimbrough L, Lewis-Hardy R, et al. Social network methods for endemic foci of syphilis: a pilot project. Sex Transm Dis 2000;27:12-18.

3 Day S, Ward H, Ison C, et al. Sexual networks: the integration of social and genetic data. Soc Sci Med 1998;47:1981-92.

4 Ward H, Day S, Weber J. Risky business: health and safety in the sex industry over a 9 year period. Sex Transm Inf 1999;75:340-3.

Editor's note: See also paper by Scoular et al, p 340-3.

\section{Destigmatising STIs: remaining challenges, new opportunities}

The potential barriers to attendance at specialist sexually transmitted infection (STI) services have long been recognised. The Royal Commission report in 1916 advised that to be effective, services needed to be "skilled, free . . . and provided at the earliest possible moment." In addition, clinicians needed to be aware of "the fear of disgrace and the consequent desire for concealment" that could hamper treatment delivery. ${ }^{1}$ In many respects the UK GUM services have risen to these challenges. The majority of clinics provide timely, effective care from easy to access and well located clinics. ${ }^{2}$ We are successful at attracting new referrals and have seen a year on year increase in voluntary attendances with a record 1.5 million consultations in 1999. With this level of success it would be easy to conclude that STI services are both accessible and acceptable for at least the majority of the UK population. However, it would appear that many patients with known or suspected STIs are still reluctant to attend genitourinary medicine (GUM) clinics for care. The principal suspected reason for this is the stigma associated with an STI diagnosis, which may be reinforced by the need for attendance at GUM clinics. ${ }^{3}$ In this regard, genitourinary medicine has much in common with mental health and cancer services. However, GUM specialists particularly value open access and strive to provide a confidential, non-judgmental, and supportive service, so it is particularly galling for them that a visit to a GUM clinic should be viewed as stigmatising by many potential users.

In looking at the possible consequences of stigma on health seeking behaviour it is helpful to look at its constituent components. It is generally considered that two elements exist- "felt" stigma, which is determined by an individual's background, education, and personal experiences and "enacted" stigma, the direct consequence of those around them discovering their problem, and resulting in discrimination. The levels of stigma attached to a particular behaviour or illness are not fixed; they vary between cultures and historically. There is some evidence that within modern culture, those conditions, which are to some extent behaviour related, attract most stigma. Importantly though, both felt and enacted stigma are amenable to public education campaigns. ${ }^{4}$

In this issue of Sexually Transmitted Infections (p 340) Scoular et al examine the experience and evolution of stigma among young women recently diagnosed with chlamydial infections initially outside a GUM setting. This is an important paper because although it is recognised that the stigma associated with GUM clinics is a barrier to patient access, the question of how modern GUM services can work to modify individuals' experiences of stigma associated with STIs has been largely ignored. Using a qualitative approach with semistructured interviews the authors provide a useful insight into how people feel about being diagnosed with an STI and their experience attending a GUM clinic. These participants had often not disclosed their intended visit to others and fear, isolation, and secrecy pervaded the consultation. Gratifyingly, although a GUM clinic visit was experienced as a stressful event, these study patients did not have their worst fears recognised and some even looked upon it as a positive experience. A process of normalisation and acceptance of information provided by clinic staff resulted in these young women overcoming initial reservations about attending a GUM clinic.

The second important finding of this paper was a comparison of how these individuals viewed GUM services and a family planning clinic (FPC). The GUM clinic was viewed as more "dangerous" than family planning services, which had a more benign appeal. The FPC was valued because it appeared to be associated with a perceived distance from a "disease model" of health care. There was no embarrassment or shame associated with the FPC; however, the GUM clinic was seen as being only attended by individuals who possessed traits that were deemed to be socially and morally unacceptable. It was "normal" for young women to attend an FPC but a GUM clinic existed for "others."

So what are the challenges and tasks for those working in GUM services and those responsible for directing policy? A role of the GUM physician in the education of other healthcare professionals and the general population seems to be a key issue in destigmatising GUM clinics. The respondents in this study had very little knowledge or understanding of STIs and the scale and difficulty of the task should not be underestimated. Previous public health strategies focusing attention on "high risk" individuals have probably intensified the marginalisation and stigma associated with STIs and GUM clinics. Ignorance of STIs and the role of genitourinary medicine are not confined to the lay public and pervade many areas of health care. Many workers in frontline services are reluctant to suggest to their patients that they attend a GUM clinic in case the patient is offended at the inference. The Monks report has gone some way to address stigma by moving clinics from obscure areas of the hospital into the centre of the main hospitals and currently we have unique opportunities to 
educate others about the role and place of GUM services. ${ }^{5}$ The recent Department of Health chlamydia pilot study has shown that the rates of chlamydial infection are almost identical in those 19-24 year olds attending a GUM clinic as those asymptomatic 19-24 year olds attending a GP surgery with an unrelated problem. ${ }^{6}$ STIs can no longer be viewed as the special problems of self referrers to GUM clinics, and will have to be seen as an important component of primary health care. The introduction of universal HIV antibody testing into antenatal clinics will undoubtedly raise awareness of STIs in both pregnant women and antenatal clinic staff. ${ }^{7}$ We need to capitalise on these events and use them to break down the prejudices that exist for individuals with STIs and GUM clinics. Healthcare workers will need to develop skills in raising the subject of sexual health, in communicating the presence of disease and the need for effective management-probably involving referral to genitourinary medicine services. Clinicians will need to be able to communicate the positive nature of an early diagnosis and the ease with which STIs can be acquired, without losing sight of the opportunities in reducing risk of further disease acquisition. In addition, closer working and some integration across different sexual health services may provide choice for patients unwilling to attend traditional GUM services.

We should feel encouraged that the experience for an individual visiting a GUM clinic is generally positive, and not as bad as is often anticipated. However, we must endeavour that as our service becomes stretched and increasing demands are made on our time, we do not allow an individual to feel let down by the service we offer. Giving patients adequate time in the clinic setting to explore their feelings and to help unravel the felt stigma they may have developed must be an essential part of our job.

The surest way to end stigma related to STI is to find effective means of identifying, eradicating, and preventing infection. Until that time we must acknowledge the importance of an all too human response to circumstances.

ELIZABETH FOLEY RAJ PATEL

Department of Genitourinary Medicine, Southampton University

Hospitals NHS Trust, Southampton, Hampshire. SO14 OYG, UK

1 Royal Commission on Venereal Diseases. Final report of the Commissioners. London: HMSO, 1916 (Cmnd 8189).

2 Foley E, Patel R, Green N, et al. Access to genitourinary medicine clinics in the United Kingdom. Sex Trans Inf 2001;77:12-14.

3 Holgate HS. Some peoples' psychological experiences of attending a sexual health clinic and having a sexually transmitted infection. Roy Soc Health 1998;118:94-6.

4 Scrambler G. Stigma and disease: changing paradigms. Lancet 1998;352: $1054-5$

5 Monks working party. Report of the working group to examine the workloads in genitourinary medicine clinics. London: Department of Health, November 1988.

6 Tobin JM, Harindra V, Tucker L. The future of chlamydia screening. Sex Trans Inf 2000;76:233-4.

7 Health Services Circular. Reducing mother to baby transmission of HIV. London: NHS Executive, 1999 (HSC 1999/183).

\section{Oral sex and HIV transmission}

It is well established that oral sex may lead to the transmission of a wide variety of STIs, including HIV. ${ }^{1-4}$ As discussed elsewhere in this issue (see syphilis symposium, pp 309-26) oral sex appears to be important in the resurgence of early infectious syphilis in the United Kingdom. Many of these latter cases have been in HIV positive individuals and it is likely that co-infection with syphilis would increase the risk of (oral) transmission of HIV - as has been shown similarly in numerous studies of genital HIV/STI co-infection.

Despite recognising that transmission does occur, some feel that the underlying risk of HIV transmission via oral sex is so low as to be negligible. However, two recent studies (as yet unpublished in peer review journals) suggest that oral sex may be contributing to a higher proportion of new HIV infections than previously thought. In the first study, of 102 men who had recently seroconverted, eight $(7.8 \%)$ were attributed to oral sex. Of these eight, unprotected oral sex was the only risk factor in four, but four had also had protected anal sex. ${ }^{5}$ A second study from my own unit was of $494 \mathrm{HIV}$ positive patients (mostly homosexual) who completed a questionnaire on sexual behaviour. Six per cent believed themselves to have been infected because of oral sex alone. Further follow up of these and other patients in our unit, who believe themselves to have been infected by unprotected oral sex is ongoing and about half, where data are available, have had recurrent infections of the mouth, which could have increased their risk. ${ }^{6}$

A third report of two studies from Australia, gave contradictory results. An interview study found that a similar proportion, seven of 75 (9.3\%), homosexual men gave receptive oral intercourse as the likely source of their infection. However, the investigators felt that they must have had other risk factors as they denied ejaculation as part of their oral sex. Furthermore, in a cohort study of over 700 men, $26 \%$ reported unprotected receptive oral intercourse with ejaculation but they did not have an increased risk of seroconversion ${ }^{7}$ (for further discussion, see $\mathrm{CDR}^{8}$ )

Finally, the press release from a very recent report of an ongoing study of homosexual men from San Francisco states that receptive oral intercourse with ejaculation was a very low risk. One seroconversion was found but thought to have occurred outside the study period. However, the study population was small (198), only $20 \%$ of these claimed to have had receptive oral sex with an HIV positive partner, only $40 \%$ to ejaculation, and follow up was for only 6 months. It is therefore unlikely such a study would have had sufficient power to detect transmission, or to reject the hypothesis that transmission does occur (presented by Dr Kimberly Page-Shafer et al, National HIV prevention conference, Atlanta, August 2001).

In June 2000, the Department of Health, following the deliberations of a working party of the chief medical officers' expert advisory group on AIDS (EAGA), published a document entitled "Review of the evidence on the risk of HIV transmission associated with oral sex." "The authors concluded, as with other extensive reviews, that oral transmission of HIV occurs and that certain factors might increase the risk. These include receptive oral intercourse (ROI) with ejaculation, high viral load, and various factors which might breach the oral defence mechanisms. Saliva is protective and has a number of antiviral components, such as thrombospondin and secretory leucocyte protease inhibitor (SLPI), but these are likely to be overcome by the volume effects of seminal fluid. ${ }^{10}$

Although most of the several dozen case reports to date have been of receptive oral intercourse, it should be noted 
that there have been reports of HIV transmission associated with insertive fellatio as well as two reports of transmission associated with cunnilingus and one of insertive anilingus. ${ }^{9}$ The presence of inflammation in the mouth, caused by sores, trauma, or infection is described in some of these reports. The relative rarity of cases of HIV infection attributed to oral transmission is likely to be influenced by the rarity with which oral exposure has occurred without other forms of penetrative sexual contact and the tendency of attributing HIV transmission to any higher risk exposure which can be identified.

In recent years, many participants in studies have indulged in protective anal and vaginal sex but oral sex has normally been unprotected. This might explain why a real (but low) risk of unprotected oral sex is now becoming more apparent. Another difficulty is the power of studies to identify such a small increase in risk. For example, in one important cohort study from four sites, the multivariate analysis showed that for the pooled data, the odds ratio for receptive oral intercourse and increased risk of HIV transmission was only 1.01 (95\% CI $1.00-1.02) .{ }^{11}$

The dilemma is how to present this small but real risk as appropriate public health messages. Concern has also been raised that highlighting the risk of unprotected oral sex may incite higher risk sexual practices as alternatives. Conversely, it is important that individuals and the public understand that oral sex is not risk free and may lead to transmission of HIV as well as other STIs.

UNAIDS and Centers for Disease Control (CDC) state, on their websites, that a condom or dental dam is recommended to reduce the risk of HIV transmission when indulging in oral sex. The expert advisory group on AIDS, following the publication of the working party review on the evidence on the risk of HIV transmission and oral sex last year, recently released a statement on risk. This reads as follows:

"There is a risk of HIV transmission during unprotected oral sex. This risk is less than from unprotected penetrative anal or vaginal sex. The risk of HIV and other sexually-transmitted infections can be reduced by using a condom for all forms of penetrative sex, including oral sex. If a condom is not used, avoidance of ejaculation into the mouth probably lessens (but does not eliminate) the risk of HIV transmission."

This risk statement recognises that oral sex is often unprotected, despite official recommendations, and enters into the discussion of what other factors might reduce the risk. A more comprehensive discussion, in the form of questions and answers, is available on the Department of Health website: www.doh.gov.uk/eaga. A recent issue of the Communicable Disease Review (CDR) has also discussed oral sex, as has National AIDS Manual (NAM), which has provided a useful fact sheet. ${ }^{8}{ }^{12}$ The Terence Higgins Trust has also relaunched its "Use your head" campaign, avoiding the use of the word "rare" in describing the risk of oral sex as this may be misinterpreted and equated with negligible risk.

Are there figures to assist counselling of the risk of oral sex on an individual level? Samuel et al, using several different mathematical models, estimated a per partner risk for receptive oral intercourse at about $1 \%$ (range $0.85-2.3 \%$ ) where per partner relates to the risk with that partner, uncontrolled for sexual activity. ${ }^{13}$ It is of course the per contact risk that we need to consider when approaching the contentious issue of post exposure prophylaxis (PEP) following sexual exposure. Is there ever any justification for using PEP following oral sex? There have been no per contact risks provided for unprotected receptive fellatio with a known HIV positive individual. However, Vittinghoff et al have come up with an estimate of $0.04 \%$ following receptive oral intercourse with a known, or possibly HIV infected, partner. ${ }^{14}$ Clearly, there may be factors which might increase this overall risk and, as always with PEP, either following occupational or sexual exposure, an individual risk assessment needs to be performed. ${ }^{15}$ Receptive fellatio with ejaculation with a known HIV individual is probably the only oral sex activity of sufficient risk to justify consideration of provision of PEP. Additional factors such as a known high viral load in the source, recent dental surgery, pharyngitis, trauma, oral ulceration, or bleeding gums would also increase the likely risk. Clearly if the patient requesting PEP regularly has unprotected receptive oral intercourse with known HIV positive individuals then counselling him/her around this behaviour would probably be more important than provision of PEP.

In conclusion, unprotected oral sex carries a risk for the transmission of HIV. Owing to the frequency with which it is practised and given the fact that those with the highest risk of acquiring HIV often have protected anal or vaginal sex, it is possible that it may lead to $6-8 \%$ of new HIV infections. Although using a condom will reduce the risk of transmission of HIV and other STIs, following penetrative oral sex, it has to be recognised that many will choose not to follow that advice. A wider discussion of risk assessment should take place so that individuals might make informed choices about their sexual behaviour.

DAVID A HAWKINS

HIV/GU Medicine Directorate, Chelsea and Westminster Hospital,

London SW10 9NH, UK

david.hawkins@chelwest.nhs.uk

1 Edwards S, Carne C. Oral sex and the transmission of viral STIs. Sex Transm Inf 1998; 74:6-10.

2 Edwards S, Carne C. Oral sex and the transmission of non-viral STIs. Sex Transm Inf 1998;74:95-100.

3 Rothenberg RB, Scarlett M, del Rio C, et al. Oral transmission of HIV. AIDS 1998;12:2095-105.

4 Robinson EK, Evans BG. Oral sex and HIV transmissions. AIDS 1999;13: 737-8.

5 Dillon B, Hecht FM, Swanson M, et al. Primary HIV infection associated with oral transmission: the Options Project UCSF. 7th Conference on Retroviruses and Opportunistic Infection, San Francisco, 2000 (abstract 473)

6 Khan AW, Richards CM, Mandalia S, et al. Safer sex in HIV infected patients in London-practices and risks. Sex Transm Inf (in press).

Grulich AE, Prestage G. Oral sex as a risk factor for HIV: a review of Australian data. HIV/AIDS and Related Diseases Social Research Conference, Sydney, May 2000

8 CDSC. Orogenital contact (oral sex) and transmission of HIV and other sexually transmitted infections. Commun Dis Rep CDR Wkly [serial online] 2001 [cited 5 July 2001];11(1).

9 Department of Health. Report of a Working Group of the UK Chief Medical Officers' Expert Advisory Group on AIDS. Review of the evidence on the risk of HIV transmission associated with oral sex. June 2000.

10 Cohen MS, Shugars DC, Fiscus SA. Limits on oral transmission of HIV-1. Lancet 2000;356:272.

11 Page-Shafer K, Veugelers PJ, Moss AR, et al. Sexual risk behaviour and risk factors for HIV-1 seroconversion in homosexual men participating in the factors for HIV-1 seroconversion in homosexual men participating in the Tricontinen.

12 National AIDS Manual. AIDS Treatment Update, 103. Factsheet no 55. NAM, July 2001

13 Samuel MC, Hessol N, Shiboski S, et al. Factors associated with human immunodeficiency virus seroconversion in homosexual men in three San Francisco cohort studies, 1984-1989. F Acquir Immune Defic Syndr 1993;6: 303-12.

14 Vittinghoff E, Douglas J, Judson F, et al. Per-contact risk of human immunodeficiency virus transmission between male sexual partners. Am f Epidemiol 1999;150:306-11.

15 Hawkins DA. Postexposure to HIV prophylaxis. Curr Opin Infect Dis 2000; $13: 3-57$ 